Mike Rumpeltes, Dagmar Höfferer-Brunthaler, Christina Schweiger, Ingrid Krottendorfer, Bernadette Pfeiffer

NCoC für Kulturelle Bildung, Pädagogische Hochschule Niederösterreich

\title{
National Center of Competence für Kulturelle Bildung
}

\author{
Netzwerk „Tanz in der Schule“
}

DOI: https://doi.org/10.53349/sv.2021.i3.a122

Das National Center of Competence ( $\mathrm{NCOC}$ ) für Kulturelle Bildung hat als eine seiner Hauptaufgaben die Entwicklung und Durchführung österreichweiter Fortbildungsveranstaltungen, denen die kulturelle Bildung in der Schule als umfassendes Bildungsprinzip zugrunde liegt. Den schon seit vielen Jahren erfolgreich existierenden Netzwerken Musik, Bildnerische Erziehung, Werken und Theater wurde seit dem Schuljahr 2019/20 das Netzwerk „Tanz in der Schule“ hinzugefügt.

Würden wir mehr tanzen, die Welt wäre eine andere!

Andreas Nero Nick (*1955), deutscher Komponist und Texter

Das Unterrichtsfach „Tanz" ist an vielen österreichischen Schulen der Primar- und Sekundarstufe im Fächerkanon als Unverbindliche Übung oder vorwiegend an Schwerpunktschulen und Schulen mit Sonderformen als eigenes Wahl- oder Pflichtfach fix verankert. Bisher sind Fortbildungsveranstaltungen für interessierte Pädagog*innen, die sich speziell mit den methodischen und didaktischen Prinzipien, den neuesten Trends und deren Vermittlung beschäftigen wollen, immer im Rahmen von Seminaren für die Fächer Musik bzw. Bewegung und Sport verankert gewesen.

Aus diesem Grund wurde durch die Initiative von Mike Rumpeltes (NCoC, PH NÖ) und Astrid Weger (Mozarteum Salzburg, Musisches Gymnasium Salzburg) im Schuljahr 2019/20 die Idee geboren, am NCoC für Kulturelle Bildung neben den schon seit vielen Jahren bestehenden Netzwerken Musik, Bildnerische Erziehung (BE), Werken und Theater eine neue Community Netzwerk „Tanz in der Schule“ zu etablieren. Die Aufgabe des neuen Netzwerkes soll die ös- 
terreichweite Vernetzung der Tanzpädagog*innen sowie die Entwicklung und Durchführung von Fortbildungsveranstaltungen speziell für die Bedürfnisse dieser Community sein.

\section{Erstes Bundesseminar Netzwerk „Tanz in der Schule“}

Als fixe jährliche Veranstaltung ist ein zweitägiges Bundesseminar Netzwerk „Tanz in der Schule" im Kalender des NCoC eingeplant. Wie so viele Veranstaltungen konnte dieses Seminar im Frühjahr 2020 jedoch coronabedingt nicht stattfinden. Die Fortbildungsveranstaltung mit dem Titel „Think Dance! Tanzen - Tanz gestalten - Tanz verstehen“ wurde um ein Jahr, auf Jänner 2021, verschoben. Trotz der zu dieser Zeit immer noch geltenden strengen Coronaregeln konnte dieses erste Bundeseminar Netzwerk "Tanz in der Schule“ in hybrider Form am Orff-Institut in Salzburg live stattfinden. Jede*r der neun renommierten Referent*innen konnte somit vor Ort in Kleingruppen mit Tanzstudierenden des Orff-Instituts den Workshop durchführen. Aufgrund ihrer Ausbildungssituation durften die Studierenden am Liveunterricht teilnehmen.

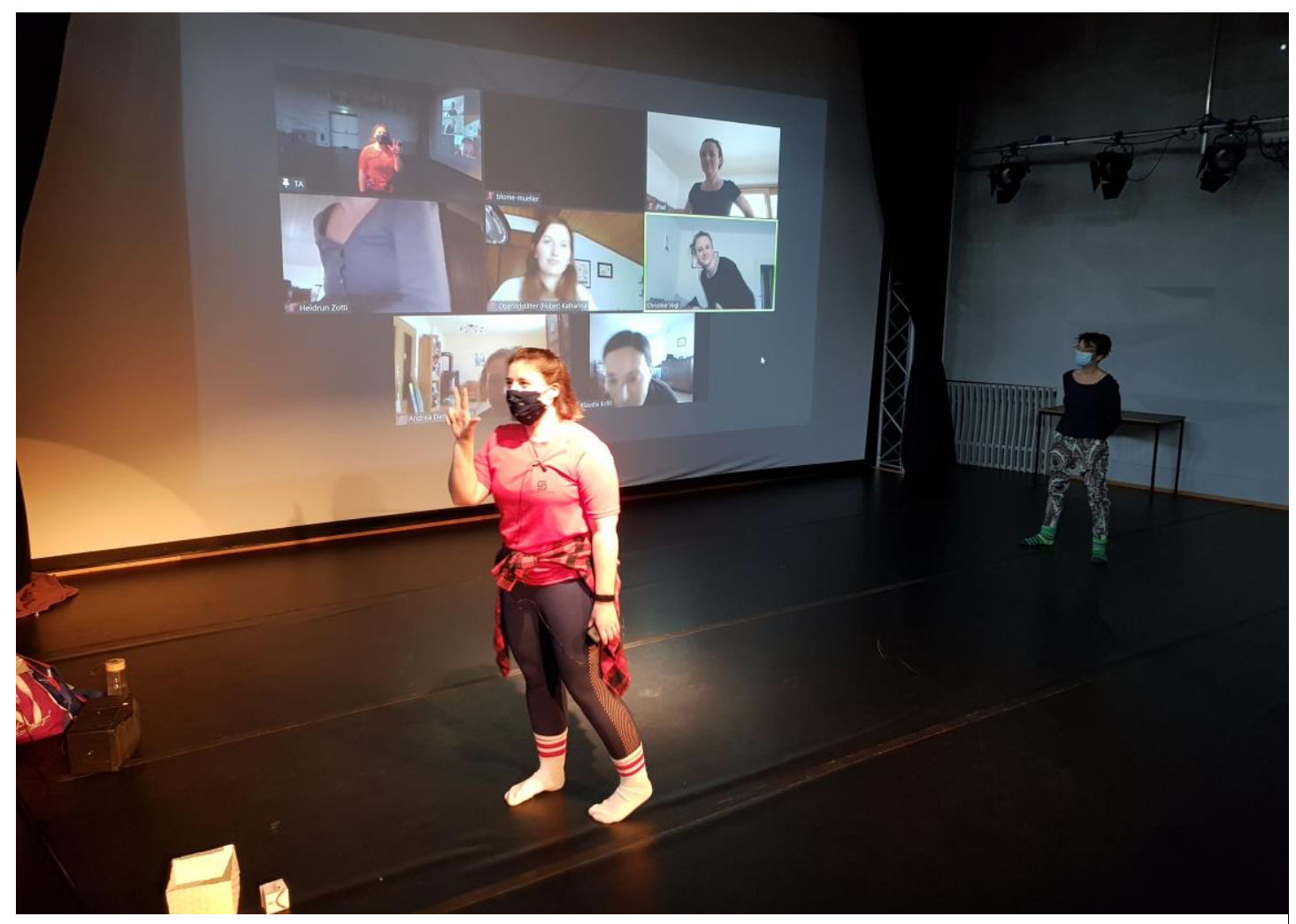

Hybrid-Workshop am Bundesseminar NW Tanz in der Schule | Foto: Mike Rumpeltes

Die über vierzig tanzbegeisterten Teilnehmer*innen aus ganz Österreich, vorwiegend Pädagog*innen aus dem Fächerkanon Musik, Tanz, Bewegung und Sport sowie Theater waren 
online von zu Hause oder aus dem Bewegungsraum einer Schule über eine Meeting-Plattform zugeschaltet und konnten bei den Workshops selbst mitmachen.

Eine große Herausforderung stellte die technische Umsetzung dieser Hybridveranstaltung dar. Das zeigte sich schon bei der Seminareröffnung, wo eine Referentin via Video aus Berlin zugeschaltet war.

Unterricht mit Mikrofon und Kamera, mit Maske und gebotenem Abstand tanzen, über Beamer die Teilnehmer*innen dazugeschaltet, das alles war auf der einen Seite eine technische Glanzleistung der Techik-Crew am Orff-Institut und auf der anderen Seite eine große Herausforderung für die Referent*innen und Teilnehmer*innen, die trotz dieser Hybridlösung bis zur letzten Minute mit Eifer bei der Sache geblieben sind. Der für uns Pädagog*innen zur Gewohnheit gewordene hybride oder gänzlich virtuelle Unterrichtsalltag konnte auch hier erlebt werden. Alle Workshopleiter*innen passten ihren Inhalt und ihre Methodik hervorragend dieser Unterrichtsform an und reagierten auf die digitalen Herausforderungen ebenso professionell wie auf alle tänzerischen Fragen, die im direkten Kontakt mit den Studierenden und Seminarteilnehmer*innen aufgekommen sind.

Durch den deutlichen Lehrplanbezug der einzelnen Workshops bekamen die Kolleg*innen Einblicke in unterschiedliche Methoden sowie Inspirationen und Ideen für den eigenen Unterricht. Einige der Workshops waren allgemein für alle Schultypen und Alterstufen der Schüler*innen gehalten, andere wiederum sprachen entweder die Primarstufe oder die Sekundarstufe an (https://www.ncoc.at/netzwerke/tanz).

Die breite Vielfalt der Workshops spannte sich von „Creative Dance 4 all“ (Alicia Kidman - Primar-/Sekundarstufe) , "Contemporary Dance Choreography“ (Kun-Chen Shih - Sekundarstufe), „Ein Fisch - oder doch eine Katze?" (Andrea Ostertag - Sekundarstufe) über Laban works @ School Lecture (Astrid Weger-Purkhart - Sekundarstufe), , Von der Bewegung zum Theaterspielen“ (Gerald Schwarz - Sekundarstufe) und „Body- and More-Percussion zu Coronazeiten“ (Nina Minimayr - Primar-/Sekundarstufe) bis hin zu „DDD - Drama Disco Dancing“ (Alicia Kidman - Primar-/Sekundarstufe).

\section{Netzwerk „Tanz in der Schule“ - österreichweite Vernetzung}

Ein wesentlicher Bestandteil der Bundesseminare aller Netzwerke am NCoC für Kulturelle Bildung der PH Niederösterreich ist die Möglichkeit der Vernetzung. Diesem Schwerpunkt, der die NCoC-Fortbildungsveranstaltungen besonders prägt, wird zeitlich dementsprechend Raum gegeben.

Nicht nur die Covidsituation an den einzelnen Schulen, sondern die schultypischen oder standortspezifischen Bedingungen, unter denen Tanz unterrichtet werden kann, waren in diesen Vernetzungsgesprächen am Bundesseminar im Jänner 2021 Diskussionsthema.

Zwei virtuelle Vernetzungstreffen (schultypenspezifisch sowie nach Regionen) wurden im Rahmen des Seminars angeboten und auch intensiv zum Austausch genutzt. In allen Kleingruppen war die Energie und der Enthusiasmus aller Lehrenden für Tanz in der Schule spürbar, es machte sich durch die Gründung dieser Community eine euphorische Aufbruchstimmung breit. Zu folgenden vorgegebenen Themen wurde diskutiert: 
- Tanz als Kultur und Haltung verstehen und transportieren.

- Formate, Möglichkeiten und Anerkennung schaffen.

- Standards und Kompetenzraster in Schule und Ausbildung sichtbar machen.

- Die Kraft der Teambildung durch Tanz hervorheben.

- Die positive Entwicklung von Persönlichkeit und Bewegungsvokabular darlegen.

- Überzeugungsarbeit gegenüber der Schulleitung leisten.

- Aus- und Fortbildungsmöglichkeit für Tanzpädagog*innen intensivieren.

Diese vielfältigen Diskussionspunkte und Themenbereiche bilden die Basis für zukünftige Themenschwerpunkte des Bundesseminars Netzwerk „Tanz in der Schule“.

Das erste Bundesseminar Netzwerk „Tanz in der Schule“ wurde am ersten Abend durch ein virtuelles Rahmenprogramm abgerundet. Wo man sich normalerweise zu Nichtcoronazeiten in Präsenz zu einem Live-Event zusammenfindet und anschließend bei einem lockeren Gespräch Kolleg*innen kennenlernen und sich mit ihnen austauschen kann, zeigten dieses Mal drei Tanzsolistinnen des Musischen Gymnasiums in Salzburg unter der Leitung von Astrid Weger ihre im Unterricht entstandenen und selbst kreierten Choreografien.

\section{Sommerworkshop Netzwerk "Tanz in der Schule“}

Einen weiteren Fixpunkt im bundesweiten Fortbildungsangebot des Netzwerkes "Tanz in der Schule" bildet ein Sommerworkshop. Das Seminarthema im Sommer 2022 wird "Masterclass Community Dance" und der Veranstaltungsort von 18. bis 20-7.2022 das Festpielhaus St. Pölten sein.

Die Schritte des pädagogisch, sozial und künstlerisch wertvollen Projekts „Community Dance (,Rhythm is it!')" werden in dieser Masterclass eingeübt, erprobt und präsentiert. Die Teilnehmer*innen lernen Bewegungen aus dem Urban Dance und aus dem zeitgenössischen Tanz, kreative Impulse zur Bewegungsfindung mit Dynamik und Sounds sowie eine gemeinsame Choreografie. Mit diesem Werkzeugkasten für kreativen zeitgenössischen Tanzunterricht und Urban Styles werden die Teilnehmer*innen die choreografischen Erfahrungen in ihrem Unterricht anwenden können.

\section{Hochschullehrgang „Tanz in der Schule“ an der PH NO̊}

Mit großem Enthusiasmus und Engagemant arbeitet das NCoC für Kulturelle Bildung im Moment an der Ausarbeitung und Genehmigung eines bundeweiten Hochschullehrgangs „Tanz in der Schule" für alle interessierten Kolleg*innen aus dem Primar- und Sekundarstufenbereich. Die Fortbildung wird 3 Module (Semester) mit insgesamt 15 ECTS umfassen und soll im WS 2022/21 starten.

Der Lehrgang unterstützt Lehrende in ihrer Arbeit in Hinblick auf ihre individuelle und differenzierende Förderung von Lernenden bei der Vermittlung von Schlüsselkompetenzen wie Persönlichkeitsbildung, Begabungsförderung, gruppendynamisches und gesundheitsbewusstes Lernen, bewegungssprachliche, künstlerische und interkulturelle Bildung. Tanz in der Schule bietet Strukturen, die besonders geeignet sind für einen jahrgangs- und fächerüber- 
greifenden, projekt- und handlungs-, aufgaben- und problemlösungsorientierten, gendersensiblen und integrativen Unterricht.

\section{Autor*innen}

Mike Rumpeltes, Prof. Mag., OstR

Mitarbeiter im Kompetenzcenter für Kulturelle Bildung, Schwerpunkte Musik, Tanz und Digitalisierung. Fortbildner und Hochschullehrer an der Pädagogischen Hochschule Niederösterreich im Bereich Musik- und Kulturpädagogik. Seine Schwerpunkte liegen u.a. am dialogischkompetenzorientierten Unterrichten und der Vernetzung der Musikpädagog*innen Österreichs im Primar- und Sekundarbereich.

Kontakt: m.rumpeltes@ph-noe.ac.at

Dagmar Höfferer-Brunthaler, Mag. MA, OStR

Mitarbeiterin im Kompetenzcenter für Kulturelle Bildung, Hochschullehrende, Fortbildnerin an der Pädagogischen Hochschule Niederösterreich, Schwerpunkt: Theater in der Schule. Entwicklung von digitalen Fortbildungsformaten. Forscht im Bereich Kultureller Bildung, auch im internationalen Kontext, z.B. International Teacher-Artist-Partnership-PD.

Kontakt: dagmar.hoefferer@ph-noe.ac.at

Christina Schweiger, MMag. Dr.

Leiterin des Kompetenzcenters für Kulturelle Bildung, Hochschullehrende, Fortbildnerin an der Pädagogischen Hochschule Niederösterreich, Schwerpunkt: Bildnerische Erziehung.

Kontakt: christina.schweiger@ph-noe.ac.at

Ingrid Krottendorfer, Mag.

Mitarbeiterin im Kompetenzcenter für Kulturelle Bildung, Hochschullehrende, Fortbildnerin an der Pädagogischen Hochschule Niederösterreich, Schwerpunkte: Theater in der Schule, Leiterin der Hochschullehrgänge Kulturpädagogik und Kulturelle Bildung in und mit Museen. Kontakt: ingrid.krottendorfer@ph-noe.ac.at

Bernadette Pfeiffer, Mag.

Mitarbeiterin im Kompetenzcenter für Kulturelle Bildung, Fortbildnerin an der Pädagogischen Hochschule Niederösterreich, Schwerpunkt: Digitalisierung.

Kontakt: bernadette.pfeiffer@ph-noe.ac.at 